\title{
La Universidad en la Escuela: Proyecto de Colaboración con la Red de Centros Comunidades de Aprendizaje
}

\section{The University in the School: Project of Collaboration with the Network of Schools as Learning Communities}

\begin{abstract}
Blas Segovia Aguilar, María del Mar García Cabrera²
Fecha de recepción: 20/01/2020; Fecha de revisión: 10/02/2020; Fecha de aceptación: 25/02/2020

Cómo citar este artículo:

Segovia, B., \& García, Ma . (2020). La Universidad en la Escuela: Proyecto de Colaboración con la Red de Centros Comunidades de Aprendizaje. Revista de Innovación y Buenas Prácticas Docentes, 9(2), 24-36.

Autor de Correspondencia: bsegovia@uco.es ue1gacam@uco.es

\section{Resumen:}

En esta aportación describimos el proyecto de innovación docente "La universidad en la escuela: Proyecto de colaboración con la Red de Centros Comunidades de Aprendizaje", realizado durante el curso 2017/2018 cuya finalidad principal es fortalecer líneas de cooperación entre las escuelas de Comunidades de Aprendizaje de la provincia de Córdoba y la Facultad de Ciencias de la Educación de la Universidad de Córdoba.

Las actividades desarrolladas han implicado a profesores y estudiantes universitarios que realizan voluntariado en estos centros educativos y a docentes de los mismos, organizándose en dos escenarios: en las escuelas, participando en la aplicación de las Actuaciones de Éxito Educativo y en la Facultad, con la puesta en marcha de un Seminario de Tertulias Pedagógicas Dialógicas para la discusión teórico práctica de las bases científicas en las que se sustentan dichas Actuaciones.

Los resultados se presentan en relación a los objetivos planteados, destacando la progresiva vinculación del proyecto de Comunidades de Aprendizaje con la formación inicial del profesorado y la formación permanente de los docentes. Esto es valorado como una fortaleza que permite prolongar la dinámica de trabajo en los próximos cursos para consolidar las necesarias relaciones entre Escuela-Universidad a través de redes de cooperación mutua.
\end{abstract}

Palabras clave: actuaciones de éxito educativo, desarrollo profesional, escuelas comunidades de aprendizaje, redes de formación.

\section{Abstract:}

In this contribution we describe the result of the teaching innovation project "The university in the school: Project of collaboration with the Network of Schools as Learning Communities", carried out during the course 2017/2018 whose main purpose is to strengthen lines of cooperation between the Schools as Learning Communities of the province of Córdoba and the Faculty of Education of the University of Córdoba.

\footnotetext{
1 Universidad de Córdoba (España), bsegovia@uco.es ; CÓDIGO ORCID: http://orcid.org/0000-00031145-2099

2 Universidad de Córdoba (España), mmgarcia@uco.es ; CÓDIGO ORCID: http://orcid.org/000-00022716-8888
} 
The activities developed have involved university professors and students who volunteer in these educational centers and teachers of the schools participating in university activities. The activities have been organized in two scenarios: in the schools participating in the implementation of the Educational Success Actions and in the Faculty with the launching of a Seminary of Dialogical Pedagogical Gathering for the theoretical and practical discussion of the scientific bases on which these Actions are based.

The results are presented in relation to the objectives set, highlighting the progressive linkage of the Learning Communities project with initial teacher training and continuing professional training. This is valued as a strength that allows to prolong the work dynamics in the next courses to consolidate the necessary relations between School-University through cooperation networks.

Key Words: educational success actions, professional development, schools as learning communities, training networks. 


\section{INTRODUCCIÓN}

Desde el año 2012, la Consejería de Educación de la Junta de Andalucía señala el modelo de Comunidades de Aprendizaje como prioritario para la inclusión y el éxito educativo, tal y como se recoge en la Orden de 8 de Junio de 2012 por la que se crea la Red Andaluza "Comunidad de Aprendizaje" (Gobierno de Andalucía, 2012). En el curso 2017/2018, en torno a 100 centros de todos los niveles educativos forman parte de esta red en Andalucía, y más de 600 en el resto de España y Latinoamérica.

El proyecto de transformación de centros educativos en Comunidades de Aprendizaje se basa en la implementación de las Actuaciones Educativas de Éxito (AEE) (Flecha, 2015) y el aprendizaje dialógico (Elboj, Puigdellívol, Soler Gallart, \& Valls Carol, 2002; Aubert, Flecha, García, Flecha \& Racionero, 2008; Valls Carol \& Munté, 2010) para lograr un triple objetivo: desarrollo de la equidad, conseguir el máximo aprendizaje para el alumnado, y la consecución de una buena convivencia. Este proyecto tiene como eje fundamental la participación de la comunidad educativa (Segovia \& García-Cabrera, 2019), así como de distintas instituciones, entre ellas la universidad (Junta de Andalucía, 2012).

Los centros educativos de esta red en Andalucía han decidido su transformación global en Comunidades de Aprendizaje y han comenzado a dar pasos hacia este modelo escolar, lo que implica trabajar con la incorporación progresiva de:

- Introducción de Actuaciones Educativas de Éxito (AEE) que dan mejores resultados en cuanto al aumento del aprendizaje instrumental y la mejora de la convivencia, según evidencian las investigaciones como el Proyecto INCLUD-ED (2006-2011) y la comunidad científica internacional (INCLUDED Consortium, 2011; Flecha, 2015). Estas actuaciones son aquellas que consiguen una mejora del rendimiento académico del alumnado implicado en las mismas, mejorando las relaciones sociales de los diferentes grupos humanos implicados en el aprendizaje. Cumplen, asimismo, con dos características esenciales como son su universalidad y su transferibilidad, es decir, funcionan en todos los contextos de carácter educativo. Las AEE fueron definidas en el proyecto INCLUD-ED (INCLUD-ED Consortium, 2011), un proyecto integrado de la prioridad 7 del VI Programa Marco de la Comisión Europea que es el único de carácter educativo que se seleccionó en dicho programa.

- Prácticas educativas inclusivas que superan las prácticas segregadoras. En este sentido es determinante la participación de los familiares y otros agentes de la comunidad educativa en actividades de aprendizaje dentro y fuera del aula y la participación en la toma de decisiones de los familiares conjuntamente con el profesorado, voluntariado y alumnado a través de comisiones específicas (García \& Ríos, 2014)

- La introducción del aprendizaje dialógico Aubert, Flecha, García, Flecha \& Racionero, 2008, de cara a ir superando: 1) las jerarquías inhibidoras del diálogo; 2) las expectativas negativas; 3) las resistencias a la transformación; 4) el fracaso escolar; 5) la violencia; 6) la insolidaridad y 7) las discriminaciones racistas y sexistas.

El modelo de Comunidades de Aprendizaje está además recomendado por la Comisión Europea como un modelo a seguir para la superación de las desigualdades y el abandono escolar temprano (recomendaciones de la Comisión Europea y resoluciones de la UE) (The Council European Union, 2010). 
La vinculación de la Universidad en las Comunidades de Aprendizaje se produce en diversos planos como se observa en las experiencias que se vienen desarrollando en nuestro país (Flecha, Racionero, Tintoré \& Arbós, 2014), pues en su inicio el proyecto se gestó en la década de 1990 desde el grupo CREA (Community of Research on Excellence for All). A lo largo de 30 años se han ido creando redes de escuelas en Europa y Latinoamérica que trabajan este modelo en contacto con redes universitarias. En la actualidad, la Red Andaluza de Comunidades de Aprendizaje se compone de casi un centenar de centros de diversa tipología.

Durante el curso 2011/2012 profesores del Departamento de Educación de la Universidad de Córdoba, desde el Aula de Mejora Educativa de la UCO (AME) ponen en marcha el proyecto de innovación docente de la UCO "Estrategias de aprendizaje dialógico para la formación inicial del profesorado: colaboración con el programa "Comunidad de Aprendizaje CEIP Albolafia" (Segovia \& García, 2012) a partir del cual se establecen líneas de colaboración con las escuelas Comunidades de Aprendizaje de Córdoba en la implementación del proyecto a través de diferentes acciones: a) captación y formación de voluntariado universitario, b) formación y asesoramiento del profesorado de estos centros y c) intercambio de experiencias en el contexto universitario con la participación de maestros y maestras de las escuelas (Segovia, 2017). En consecuencia, esta relación con las escuelas Comunidades de Aprendizaje favorece acciones destinadas a incluir en los planes de estudio de los grados (Educación Infantil, Educación Primaria y Educación Social) y de postgrado (Máster Universitario en Educación Inclusiva: asignatura "Las Comunidades de Aprendizaje como estrategias de mejora socioeducativa") el estudio de este modelo escolar, y favoreciendo la participación de los docentes de los centros Comunidades de Aprendizaje en las aulas universitarias.

Por otro lado, hemos de mencionar las actuaciones destinadas a la formación del profesorado en la fase de sensibilización con la finalidad de conocer los fundamentos del aprendizaje dialógico y las pautas para implementar las prácticas educativas en los proyectos de los centros educativos que deciden iniciar el proyecto de transformación. En este caso colaborando el profesorado universitario con la Consejería de Educación del Gobierno de Andalucía (García \& Segovia, 2014; Segovia, 2017).

Todas estas acciones favorecen el desarrollo de un diálogo entre la teoría y la práctica educativa basado en la cooperación inter-institucional que repercute directamente en la formación del alumnado universitario y posibilitan la transferencia de la investigación educativa para la mejora de las escuelas.

En resumen, podemos concluir que en este proyecto se establecen dos líneas prioritarias de acción:

a) Participación del profesorado de las escuelas en la Facultad de Ciencias de la Educación. Esta modalidad de actividades implica la colaboración de los maestros y maestras en actividades en las asignaturas implicadas de titulaciones de Educación Infantil, Educación Primaria, Educación Social y Máster universitario en Educación Inclusiva y otras de difusión del proyecto en ámbito universitario. 
b) Participación del profesorado y alumnado universitario como voluntariado en la implementación de las Actuaciones Educativas de Éxito en las escuelas o en actividades encaminadas a la formación continua.

El profesorado no universitario implicado en el proyecto pertenece a los centros Comunidades de Aprendizaje: CEIP Albolafia (Córdoba), CEIP Duque de Rivas (Córdoba), IES Sácilis (Pedro Abad), CEIP Los Alcalá Galiano (Doña Mencía) y CEIP Maestro Enrique Asensi (Puente Genil). Los profesores universitarios son del Departamento de Educación y forman parte del Aula de Mejora Educativa de la UCO.

\section{DESARROLLO DE LA EXPERIENCIA DE INNOVACIÓN}

\subsection{Objetivos}

Los objetivos propuestos se han dirigido a fortalecer una red formativa entre profesorado de las escuelas que desarrollan el modelo de Comunidades de Aprendizaje y la Facultad de Ciencias de la Educación en base al desarrollo de un modelo dialógico y cooperativo.

Entre los objetivos destacamos:

1. Incentivar la participación y colaboración del profesorado de centros educativos "Comunidades de Aprendizaje" en la formación del alumnado de las titulaciones de Educación.

2. Vincular el proyecto de transformación de los centros Comunidad de Aprendizaje a la formación inicial del profesorado y los educadores.

3. Sensibilizar al alumnado universitario de la importancia de la participación en proyectos de Comunidades de Aprendizaje desde el voluntariado, como espacio privilegiado de experiencia pre-profesional.

4. Comprender los fundamentos epistemológicos del aprendizaje dialógico y las principales características de las Comunidades de Aprendizaje.

5. Formar a los y las participantes en modelos de actuación socio-comunitaria en contextos de actuación educativa preferente.

6. Participar en las actividades de formación y evaluación que se diseñen en los centros Comunidad de Aprendizaje

7. Asesorar al profesorado, familiares y voluntariado en las estrategias de transformación en las CdA.

\subsection{Competencias desarrolladas}

Las actividades que se realizan en el proyecto implican la participación de alumnado y profesorado universitario en los centros educativos que están implementado Actuaciones Educativas de Éxito, lo que contribuye a la adquisición y desarrollo de diversas competencias profesionales. La participación de los universitarios en los contextos escolares y del profesorado de Comunidades de Aprendizaje en el contexto universitario ayuda de forma significativa al desarrollo de competencias de comunicación profesional a través de diferentes escenarios de interacción, competencias fundamentales para el desarrollo de la función docente. El alumnado universitario tiene que desarrollar estrategias comunicativas basadas en el diálogo igualitario con el profesorado de las escuelas, las familias y el alumnado, fortaleciendo de esta forma su campo experiencial. Por otra parte, el profesorado (tanto universitario como de las escuelas participantes) fortalece su argumentación pedagógica a partir de los diálogos que se mantienen en el seminario de formación y que se han suscitado a partir de los documentos trabajados. Esta cuestión resulta fundamental en la consolidación de los saberes necesarios para la práctica pedagógica y para una competencia docente en línea con las necesidades actuales de la sociedad de la 
información. Tal y como señala la investigación (Rodríguez, Condom-Bosch, Ruiz \& Oliver, 2020) este tipo de prácticas formativas tienen un gran impacto en el desarrollo profesional de los docentes, tanto inicial como permanente.

\subsection{Metodología}

La metodología de este proyecto se sustenta en la investigación-acción participativa (PAR) (Elliott, 1993; Kemmis \& McTaggart, 1992), favoreciendo la construcción de la teoría educativa en relación con la reflexión desde la práctica para su aplicación en diversas asignaturas de grado y de postgrado.

Las características más relevantes de la metodología son:

a) La construcción cooperativa del conocimiento en contextos diversos: aula universitaria, seminarios o centros educativos

b) Las prácticas inclusivas basadas en la igualdad de las diferencias y en el aprendizaje dialógico.

c) Los diferentes agentes involucrados en una realidad concreta desarrollan una intervención sistemática para transformarla y mejorarla (Sepúlveda-Ruiz, CalderónAlmendros \& Torres-Moya, 2012)

Como elemento diferenciador de la metodología que caracteriza este proyecto de innovación educativa hemos de destacar la orientación dialógica de la misma, lo que influye en la puesta en práctica del principio de diálogo igualitario (Flecha, 1997) para que las interacciones que se producen entre los maestros y maestras de las escuelas, el profesorado y estudiantes universitarios se sustenten en la fuerza de los argumentos reconociendo las aportaciones que se realizan desde el saber teórico y el saber práctico. En definitiva, creamos un espacio favorecedor de la formación profesional docente creando nexos entre la Escuela y la Universidad (García-Carrión, Gómez, Molina \& Ionescu, 2017)

\subsection{Actividades}

Se han desarrollado actividades en las que se han implicado diferentes participantes en diversos escenarios educativos, sobre todo en los centros Comunidades de Aprendizaje (voluntarios estudiantes-profesorado universitario-maestros y maestras de las escuelas y familiares y otros participantes comunitarios) y en la Facultad de Ciencias de la Educación (profesorado universitario, estudiantes colaboradores y maestros de los centros educativos). Destacamos las que siguen:

\section{JORNADAS DE VOLUNTARIADO EN COMUNIDADES DE APRENDIZAJE}

Realizadas a principio de curso con la finalidad de dar a conocer los proyectos educativos de los centros educativos y sensibilizar al alumnado universitario para que se impliquen el programa de voluntariado, que se coordina desde el Aula de Mejora Educativa de la Universidad de Córdoba. Los ponentes en las mismas son maestros y maestras de las Comunidades de Aprendizaje, voluntarios y voluntarias de cursos anteriores y profesorado del Aula de Mejora Educativa.

El programa desarrollado en esta edición ha sido el que se observa en la figura 1: 


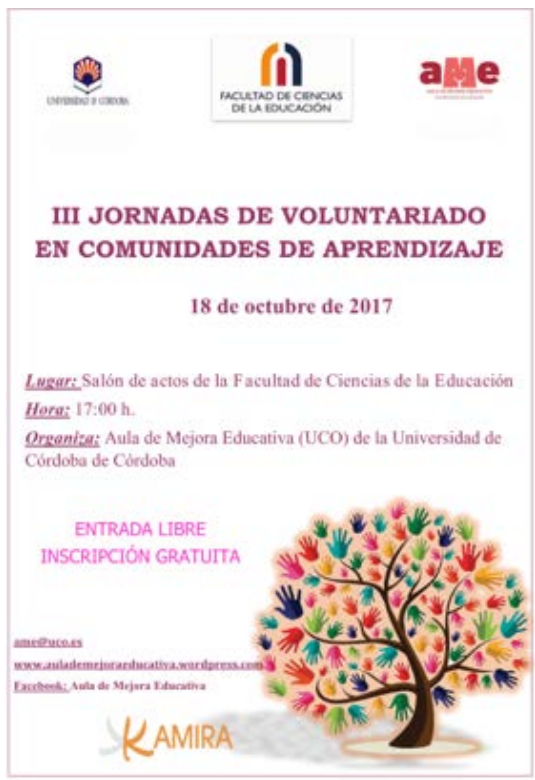

\section{Presentación y bienvenida}

Decana de la Facultad de Ciencias de la Educación

2. "La mejora escolar desde Comunidades de Aprendizaje: participación y actuaciones educativas de éxito"

Coordinador del Aula de Mejora Educativa

3. Los proyectos de las Comunidades de Aprendizaje en la provincia de Córdoba

- C.E.I.P. Antonio Gala (Córdoba) (Jefe de Estudios)

- C.E.I.P. Albolafia (Córdoba) (Director)

- CEIP Duque de Rivas (Córdoba) (Directora)

4. Actuaciones realizadas por el Aula de Mejora Educativa en los centros educativos

- Proyecto "Visitando mi futuro" (Voluntarias y alumnas de $3^{\circ}$ Educación Primaria)

- Proyecto "Miradas alternativas desde la diversidad" en

Figura 1. Cartel anunciador de III Jornadas.

CEIP Duque de Rivas (Voluntarias y alumnas de $3^{\circ}$ Educación Social)

- Proyecto "Experimenta conmigo": (Voluntarias y alumnas de Máster en Ed. Inclusiva y $4^{\circ}$ Educación Primaria)

- Proyecto de Formación de Familiares en CEIP Albolafia: (Voluntaria y alumna de Máster en Ed. Inclusiva)

- Tertulia Literaria Dialógica: "Dialogando con los clásicos" (Voluntario senior de TLD)

- Grupos Interactivos: "Acelerando el aprendizaje, mejorando las interacciones en el aula": (Voluntario y alumno de $3^{\circ}$ de E. Primaria)

5. Compromiso de voluntariado con los diferentes centros educativos para el curso $2017 / 2018$.

6. Cumplimentación de documento de solicitud de voluntariado

\section{SEMINARIO DE LECTURA DIALÓGICA PEDAGÓGICA}

Con la finalidad de profundizar en aspectos clave concernientes a las bases científicas sobre las que se sustentan las Actuaciones Educativas de Éxito que se ponen en práctica en las Comunidades de Aprendizaje se ha puesto en marcha la actividad formativa de Seminario de Tertulias Pedagógicas Dialógicas en la Facultad de Ciencias de la Educación, siguiendo el modelo de la AEE "Formación Dialógica del Profesorado". Con una secuencia de una reunión al mes se han mantenido debates siguiendo la metodología de las Tertulias Dialógicas (Valls, Soler, Flecha, 2008) en los que han participado tanto profesores y profesoras universitarios, alumnado universitario y profesorado de los centros educativos. La estructura y temáticas desarrolladas han sido:

Sesión 1. Tema: La tertulia dialógica en la formación del profesorado

Presentación

Texto: CREA. (sf) Seminario de Lectura Dialógica.

Sesión 2. Tema: Las interacciones en el aula.

Textos:

Instituto Natura (2013) Grupos Interactivos. Sao Paulo. 
García Carrión, R., Molina, S. Grande, L.A. y Buslón, N. (2016) Análisis de las Interacciones entre Alumnado y Diversas Personas Adultas en Actuaciones Educativas de Éxito: Hacia la Inclusión de Todos y Todas. Revista Latinoamericana de Educación Inclusiva, 10(1), 115-132.

Sesión 3. Tema: Socialización preventiva en violencia de género

Conferencia a cargo del profesor Ramón Flecha García. Catedrático de Sociología en la Universidad de Barcelona (Figura 2).

Texto: Instituto Natura (2014) Modelo dialógico de prevención y resolución de conflictos. Sao Paulo.

Sesión 4. Tema: La inclusión del alumnado con necesidades educativas especiales en Comunidades de Aprendizaje

Texto: Molina, S. (2015) La inclusión del alumnado con necesidades educativas especiales en las Comunidades de Aprendizaje. Intangible Capital 11(3), 272-292.

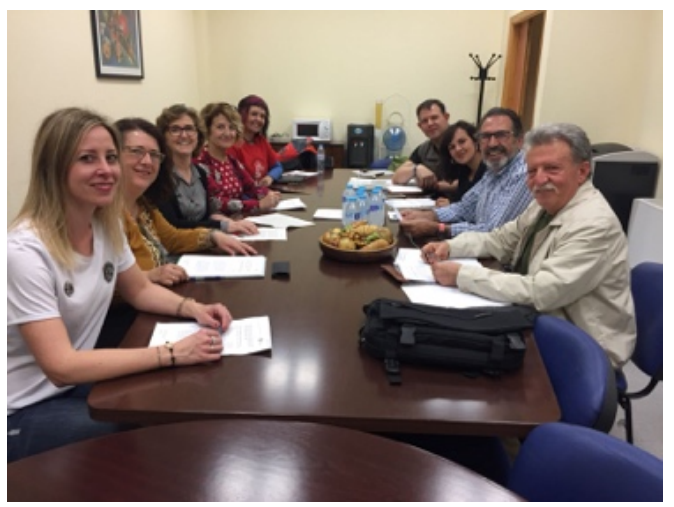

Sesión 5. Tema: La lectura dialógica y los principios del Aprendizaje Dialógico

Textos: Instituto Natura (2013) Tertulia Dialógica. Sao Paulo.

CREA, Periódico Escuela (2012) Las tertulias literarias dialógicas. Wolters Kluwers. Madrid.

Aubert, A. et al. (2010) "El diálogo igualitario" en Aprendizaje dialógico en la sociedad de la información. Hipatia, Barcelona.

Figura. 2. Seminario de Tertulias Pedagógicas Dialógicas.

\section{RESULTADOS}

Los resultados obtenidos en el desarrollo del presente proyecto de innovación docente los analizamos en función de los objetivos propuestos. Haremos una breve síntesis de los progresos y de los retos que quedan por conseguir.

\subsection{Incentivar la participación y colaboración del profesorado de centros educativos "Comunidades de Aprendizaje" en la formación del alumnado de las titulaciones de Educación.}

Hemos iniciado este tipo de actividad con la participación del director de CEIP Albolafia en la asignatura del Máster Universitario en Educación Inclusiva "Las Comunidades de Aprendizaje como estrategia de mejora socioeducativa". Las otras actuaciones se han centrado en la participación en las III Jornadas de Voluntariado en Comunidades de Aprendizaje. Por lo tanto, es un objetivo sobre el que debemos seguir trabajando, pues las posibilidades de participar en escenarios universitarios es necesaria dadas las valoraciones positivas que se desprenden de las opiniones del alumnado. 
3.2. Vincular el proyecto de transformación de los centros Comunidad de Aprendizaje a la formación inicial del profesorado y los educadores.

La participación de profesorado implicado en el proyecto viene desarrollando este objetivo desde el curso 2011-2012, de forma que la presencia y el impacto del modelo educativo de Comunidades de Aprendizaje se afianza en mayor medida en cada curso académico, lo que se ve reflejado en los contenidos de diferentes asignaturas impartidas por el profesorado del Departamento de Educación, como Planificación Educativa en Educación Infantil y en Educación Primaria.

3.3. Sensibilizar al alumnado universitario de la importancia de la participación en proyectos de Comunidades de Aprendizaje desde el voluntariado, como espacio privilegiado de experiencia pre-profesional.

Valoramos en alto grado la consecución de este objetivo y su impacto en los aprendizajes del alumnado que participa en el Programa de Voluntariado en Comunidades de Aprendizaje. El número de estudiantes participantes en el voluntariado ha variado entre 40 a 60 a lo largo del curso. Destacamos la influencia que esta experiencia ejerce en los universitarios de los dos primeros años de grado, muchos de ellos permanecen en el programa hasta finalizar sus estudios, fortaleciendo su vocación como educadores y su implicación social con los contextos más desfavorecidos.

Las solicitudes para incorporarse al programa de voluntariado tras las III Jornadas han sido de 43 alumnos y alumnas de las diferentes titulaciones de la FCE. A lo largo del curso se han ido incorporando otros voluntarios y voluntarias hasta llegar a 60. En ello ha influido la difusión de la información sobre el programa de Voluntariado en Comunidades de Aprendizaje. Según la titulación de procedencia 6 estudiantes están matriculados en Educación Infantil, 29 en Educación Primaria y 25 en Educación Social. Las cifras son equiparables a las de años anteriores. Destacamos en este curso la presencia de los estudiantes de Educación Social en proporción a la cantidad de alumnos matriculados en la misma.

Los voluntarios y voluntarias se han distribuido entre los colegios Comunidades de Aprendizaje CEIP Albolafia (Barriada Sector Sur- Calle Torremolinos), CEIP Antonio Gala (Barriada de Las Moreras), CEIP Duque de Rivas y CEIP Pedagogo García Navarro (Barriada de Las Palmeras), centros situados en contextos reconocidos por la Consejería de Igualdad y Bienestar Social como Zonas con Necesidades de Transformación Social o Zonas Desfavorecidas. Sus implicaciones en las actividades de los centros educativos han estado organizadas por los correspondientes equipos directivos siendo supervisadas por el profesorado universitario implicado en este proyecto. Tenemos constancia de estudiantes que iniciaron su actividad de voluntariado en los primeros cursos del grado y que han orientado sus estudios de postgrado en esta línea de innovación e investigación.

\subsection{Comprender los fundamentos epistemológicos del aprendizaje dialógico y las principales características de las Comunidades de Aprendizaje.}

La actividad desarrollada en el Seminario de Tertulias Pedagógicas ha favorecido de forma significativa el debate sobre los principios psicopedagógicos en los que se sustentan las AEE y las dificultades y aciertos encontrados en su aplicación práctica con alumnado de diferentes edades en los diversos centros educativos. Se han realizado cinco sesiones a lo largo del curso en las que se han debatido 8 textos de la literatura científica relacionada con Comunidades de Aprendizaje, con una participación media de 9 personas procedentes del ámbito universitario y escolar. 
Esta actividad tiene un valor sobreañadido para el profesorado de las Comunidades de Aprendizaje que han iniciado la implementación de las AEE, pues ha permitido interaccionar con otros colegas con más experiencia, profundizar y analizar la aplicación de Grupos Interactivos y Tertulias Literarias Dialógicas desde un enfoque teórico-práctico. Sin embargo, estimamos que es necesario seguir intensificando este modelo de formación continua del profesorado pues quedan muchos aspectos que tratar.

\subsection{Formar a los y las participantes en modelos de actuación socio-comunitaria en contextos de actuación educativa preferente.}

Este objetivo está orientado fundamentalmente hacia el alumnado universitario que participa en el programa de voluntariado en Comunidades de Aprendizaje.

La valoración del impacto de su participación en este programa y las repercusiones en su identidad profesional aún no se ha realizado, pues requiere de la aplicación de los cuestionarios que tenemos creados y su correspondiente análisis. Este proceso se ha iniciado.

\subsection{Participar en las actividades de formación y evaluación que se diseñen en los centros Comunidad de Aprendizaje}

La transformación de un centro educativo en Comunidad de Aprendizaje requiere de una serie de fases: sensibilización, transformación y consolidación. En esta última es importante los procesos de evaluación, junto con la formación del profesorado y la investigación.

Tangencialmente al proyecto el profesorado universitario que participa en el mismo inició el diseño de un programa de evaluación para las escuelas que deseen implementarlo con el apoyo de la Comisión Provincial de Comunidades de Aprendizaje de Córdoba. El trabajo está sin concluir, aunque están definidas las dimensiones y se han realizado o adaptado instrumentos para la evaluación de las AEE, la participación y la gestión.

\subsection{Asesorar al profesorado, familiares y voluntariado en las estrategias de transformación en las CdA.}

El proyecto de innovación ha servido para el asesoramiento del profesorado que ha participado en los seminarios y puntualmente con el alumnado voluntario en las CdA. El grado de consecución de este objetivo es relativo, pues depende del liderazgo pedagógico que los propios participantes en el seminario de Tertulias Pedagógicas mantengan en sus centros educativos. Estimamos que el impacto puede ser significativo, dado que la mayoría de los y las participantes forman parte de los equipos directivos de las Comunidades de Aprendizaje.

\section{CONCLUSIONES}

La cooperación de la universidad, especialmente la Facultad de Ciencias de la Educación de la Universidad de Córdoba, con los centros educativos de la red de Comunidades de Aprendizaje se constituye como un modelo específico regido por el principio de diálogo igualitario (Flecha, 1997; Valls, Prados \& Aguilera, 2014). Esta orientación, influenciada por la teoría de la acción comunicativa de Habermas (1989) 
determina la necesidad de construir el conocimiento teórico-práctico necesario para la mejora escolar a partir de las bases científicas y la discusión rigurosa de las prácticas que se desarrollan en los contextos escolares en base a argumentaciones válidas. En este proceso, es básico crear espacios de reflexión conjunta en los que participen tanto los profesores y profesoras universitarios como los de las escuelas e institutos en diferentes escenarios educativos: en las escuelas y en la universidad. Estas dinámicas nos permiten crear redes de docentes en las que las actividades formativas y las interacciones entre los saberes de la Escuela y la Universidad se complementan.

Los profesores y profesoras de los centros educativos Comunidades de Aprendizaje, desde el saber experiencial, nos permiten interrogarnos sobre cómo damos solución y orientaciones a problemas reales vinculados con los principios pedagógicos del modelo de Comunidades de Aprendizaje, así como la aplicación de los mismos en los diferentes contextos. Por otro lado, el profesorado universitario necesita confrontar su saber teórico con la realidad escolar. Esta es la dinámica que se ha iniciado en este proyecto de innovación docente con la puesta en marcha de la Actuación Educativa de Éxito "Tertulias Pedagógicas Dialógicas" en los seminarios que hemos venido realizando a lo largo del curso.

La participación del profesorado universitario en las escuelas proporciona un recurso de gran valor para su propia formación docente pues en esta adquieren similar importancia los saberes teóricos como las experiencias prácticas; y en el ámbito de las ciencias de la educación la solución al debate dialéctico entre la teoría y la práctica puede encontrar vías de solución en la creación y fortalecimiento de redes profesionales entre la Escuela y la Universidad (Segovia, 2017; Rodriguez et al, 2020). Además, la participación del profesorado de las Comunidades de Aprendizaje en este proyecto ayuda a fortalecer su liderazgo pedagógico en sus propios centros, pues este modelo de formación dialógica ofrece un nuevo sentido a la formación permanente, elemento clave para la consolidación del proyecto de Comunidades de Aprendizaje en su propia escuela.

Por último, destacamos un aspecto poco estudiado en la investigación académica y que percibimos de gran importancia: cómo están influyendo en el profesorado participante las relaciones socio-afectivas que se construyen entre los participantes en este tipo de redes de colaboración entre profesorado universitario y de los centros educativos.

Las debilidades detectadas están relacionadas con la dificultad de compaginar los horarios laborales con los de realización de las actividades -tanto en el ámbito universitario como en el de las escuelas- y la ausencia de directivos y profesorado de algunas de las escuelas en las actividades universitarias. Igualmente, la distancia geográfica entre las escuelas y la FCE dificulta la presencia de voluntariado y profesorado universitario en los escenarios escolares.

Como conclusión general valoramos positivamente el desarrollo del proyecto de innovación y buenas prácticas docentes siendo conscientes que la consolidación de este modelo de redes requiere del trabajo continuado de varios cursos.

\section{REFERENCIAS}

Aguilera, A., Mendoza, M., Racionero, S., \& Soler Gallart, M. (2010). El papel de la universidad en Comunidades de Aprendizaje. Revista Interuniversitaria de Formación del Profesorado, 67, 45-56.

Aubert, A., Flecha, A., García, C., Flecha, R., \& Racionero, S. (2008). Aprendizaje dialógico en la sociedad de la información. Barcelona: Hipatia.

CREA-Escuela (2012). Las tertulias literarias dialógicas. Revista Escuela Comunidades de Aprendizaje, 4. 
CREA-Instituto Natura (2013) .Grupos Interactivos. Sao Paulo: Instituto Natura.

CREA-Instituto Natura (2014). Modelo dialógico de prevención y resolución de conflictos. Sao Paulo, Instituto Natura.

Elboj, C., Puigdellívol, I., Soler Gallart, M. \& Valls Carol, R. (2002). Las Comunidades de Aprendizaje. Transformar la educación. Barcelona: Graó.

Elliott, J. (1993). El cambio educativo desde la investigación-acción (3 ed.). Madrid: Morata.

Flecha, R. (1997). Compartiendo palabras. Barcelona: Paidós.

Flecha, R. (Ed.). (2015). Successful Educational Actions for Inclusion and Social Cohesion in Europe. Springer International Publishing.

Flecha, R., Racionero, S., Tintoré, M. \& Arbós, A. (2014). Actuaciones de Éxito en la Universidad. Hacia la excelencia tomando las mejores universidades como modelo. Multidisciplinary Journal of Educational Research, 4 (2), pp.131-150.

Garcia, L. B., \& Ríos, O. (2014). Participation and family education in school: Successful educational actions. Studies in the Education of Adults, 46(2), 177191. https://doi.org/10.1080/02660830.2014.11661665

García, Ma M. \& Segovia, B. (2014) El compromiso social de la práctica universitaria: formación del voluntariado universitario en Comunidades de Aprendizaje. XIII Congreso Internacional de Formación del Profesorado. Investigar para acompañar el cambio educativo y social (pp.1235-1243). Santander: UCA.

Garcia-Carrion, R., Gomez, A., Molina, S., \& Ionescu, V. (2017). Teacher education in schools as learning communities: Transforming high-Poverty Schools through dialogic learning. Australian Journal of Teacher Education, 42(4), 44-56. https://doi.org/10.14221/ajte.2017v42n4.4

García Carrión, R., Molina, S. Grande, L.A., \& Buslón, N. (2016) Análisis de las Interacciones entre Alumnado y Diversas Personas Adultas en Actuaciones Educativas de Éxito: Hacia la Inclusión de Todos y Todas. Revista Latinoamericana de Educación Inclusiva, 10(1), 115-132.

Gobierno de Andalucía (2012). Orden de 8 de junio de 2012, por la que se regula el procedimiento de inscripción y continuidad de centros reconocidos como "Comunidades de Aprendizaje" y se crea la Red Andaluza "Comunidades de Aprendizaje." Boletín Oficial de La Junta de Andalucía, 46-59.

Habermas, J. (1989). Teoría de la acción comunicativa: complementos y estudios previos. Madrid: Akal.

INCLUD-ED Consortium (2011). Actuaciones de éxito en las escuelas europeas. Madrid: Ministerio de Educación.

Junta de Andalucía (2012). Orden 8 de junio de 2012, por la que se regula el procedimiento de inscripción y continuidad de centros reconocidos como 
"Comunidad de Aprendizaje" y se crea la Red Andaluza de "Comunidades de Aprendizaje". Boletín Oficial de la Junta de Andalucía número 16, de 28 de junio de 2012.

Kemmis, S., \& McTaggart, R. (1992) Cómo planificar la investigación-acción (1a ed.). Barcelona: Laertes.

Molina, S. (2015) La inclusión del alumnado con necesidades educativas especiales en las Comunidades de Aprendizaje. Intangible Capital, 11(3), 272-292.

Rodríguez, J. A., Condom-Bosch, J. L., Ruiz, L., \& Oliver, E. (2020). On the Shoulders of Giants: Benefits of Participating in a Dialogic Professional Development Program for In-Service Teachers. Frontiers in Psychology, 11, 5. https://doi.org/10.3389/fpsyg.2020.00005

Segovia, B., \& García-Cabrera, Mª (2012) Estrategias de aprendizaje dialógico para la formación inicial del profesorado: colaboración con el programa "Comunidad de Aprendizaje CEIP Albolafia". Recuperado de http://www.uco.es/organizacion/calidad/innovacioneducativa/images/documentos/ memorias/grupos-docentes/2011-2012/ciencias-educacion/114013.pdf

Segovia, B. (2017). La universidad y su participación en las comunidades de aprendizaje. Un compromiso para la transformación social y educativa. El Guiniguada. Revista de investigaciones y experiencias en Ciencias de la Educación, 20117/26, 34-46. doi: https://doi.org/10.20420/ElGuiniguada.2017.135.

Segovia-Aguilar, B., \& García-Cabrera, Ma . M. (2019) Participación de la Comunidad en la Escuela Inclusiva. En V. Marín \& N. Jiménez (coord.), Las didácticas inclusivas (pp. 93-100) Barcelona: Octaedro.

The council European Union. (2010). Council conclusions of 11 May 2010 On the social dimension of education and training (2010/C 135/02). Official Journal of the European Union, C 135/2 C 135/7.

Valls, R., Prados, M., \& Aguilera, A. (2014). El proyecto INCLUD-ED: estrategias para la inclusión y la cohesión social en Europa desde la educación. Investigación En La Escuela, 4(82), 31-43. Recuperado de: http://www.investigacionenlaescuela.es/index.php/revista-investigacion-en-laescuela/1088-el-proyecto-includ-ed-estrategias-para-la-inclusion-y-la-cohesionsocial-en-europa-desde-la-educacion

Valls, R., Soler, M., \& Flecha, R. (2008) Lectura Dialógica: interacciones que mejoran y aceleran la lectura. Revista Iberoamericana de Educación 2008/46, p. 71-87.

Valls Carol, R., \& Munté, A. (2010). Las claves del aprendizaje dialógico en las Comunidades de Aprendizaje. Revista Interuniversitaria de Formación del Profesorado, 67, 11-15. 